\title{
Riemerella anatipestifer M949_0459 gene is responsible for the bacterial resistance to tigecycline
}

\author{
Tao Li ${ }^{1}$, Min Shan ${ }^{1,2}$, Jing He ${ }^{1}$, Xiaolan Wang ${ }^{1}$, Shaohui Wang ${ }^{1}$, Mingxing Tian ${ }^{1}$, \\ Jingjing $\mathbf{Q i}^{1}$, Tingrong Luo ${ }^{2}$, Yonghong $\mathrm{Sh}^{1}{ }^{1}$, Chan Ding ${ }^{1}$ and Shengqing $\mathbf{Y u}^{1}$ \\ ${ }^{1}$ Shanghai Veterinary Research Institute, Chinese Academy of Agricultural Sciences, Shanghai, China \\ ${ }^{2}$ College of Animal Science and Technology, Guangxi University, Guangxi, China \\ Correspondence to: Shengqing Yu, email: yus@shvri.ac.cn \\ Keywords: Riemerella anatipestifer, M949_0459 gene, MIC, tigecycline, resistance, Pathology Section \\ Received: May 16, $2017 \quad$ Accepted: June 29, $2017 \quad$ Published: July 27, 2017
}

Copyright: Li et al. This is an open-access article distributed under the terms of the Creative Commons Attribution License 3.0 (CC BY 3.0), which permits unrestricted use, distribution, and reproduction in any medium, provided the original author and source are credited.

\section{ABSTRACT}

Based on its important role in last-line therapeutics against multidrug-resistant bacteria, tigecycline has been increasingly important in treating infections. However, mounting reports on tigecycline-resistant bacterial strains isolated from different sources are of concern, and molecular mechanisms regarding tigecycline resistance are poorly understood. Riemerella anatipestifer is a Gram-negative, non-motile, non-spore-forming, rod-shaped bacterium, which causes fibrinous pericarditis, perihepatitis, and meningitis in infected ducks. We previously constructed a random transposon mutant library using Riemerella anatipestifer strain $\mathrm{CH}_{3}$, in present study, we described that Riemerella anatipestifer M949_0459 gene is responsible for the bacterial resistance to tigecycline. Using the minimum inhibitory concentration assay, a mutant strain showed significantly increased (about six-fold) tigecycline susceptibility. Subsequently, the knocked-down gene was identified as M949_0459, a putative flavin adenine dinucleotide-dependent oxidoreductase. To confirm the resistance function, M949_0459 gene was overexpressed in Escherichia coli strain BL21, and the minimum inhibitory concentration analysis showed that the gene product conferred resistance to tigecycline. Additionally, expression of the M949_0459 gene under treatment with tigecycline was measured with quantitative real-time PCR. Results showed that the mRNA expression of M949_0459 gene was elevated under tigecycline treatment with dose range of 1-10 mg/L, and peaked at $4 \mathrm{mg} / \mathrm{L}$. Moreover, two kinds of efflux pump inhibitors, carbonyl cyanide m-chlorophenyl hydrazone and phenylalanine arginyl $\beta$-naphthylamide were tested, which showed no function on tigecycline resistance in the strain $\mathrm{CH} 3$. Our results may provide insights into molecular mechanisms for chemotherapy in combating Riemerella anatipestifer infections.

\section{INTRODUCTION}

Tigecycline, one member of the tetracyclines group called glycylcyclines, is a semisynthetic antibiotic with an expanded broad spectrum of potent activity against most of the clinically important bacterial pathogens, including many multidrug-resistant bacteria $[1,2]$. As a tetracycline analog, a change in structure of an additional N-N dimethylglycylamido (DMG) group at the $\mathrm{C}_{9}$ position of the $\mathrm{D}$ ring of minocycline enhances the binding ability of tigecycline to its ribosomal target site and eludes common tetracycline resistance mechanisms [3]. Once, tigecycline was considered to be a last-chance antibiotic against multi-drug resistant bacteria. Since 2007, tigecycline has been suggested by the Food and Drug Administration for the treatment of complicated intra-abdominal infections, skin infections, and community-acquired pneumonia. Unfortunately, the widespread use of tigecycline has led to bacterial resistance. So far, the mechanisms of tigecycline resistance have mainly been investigated in 
Klebsiella pneumonia, Escherichia coli, and Acinetobacter baumannii [4-7].

Riemerella anatipestifer ( $R$. anatipestifer), a member of the Flavobacteriaceae family of the rRNA superfamily V [8], infects young ducks and geese, causing a severe form of Riemerellosis containing fibrinous pericarditis, perihepatitis, and meningitis. Riemerellosis is one of the most detrimental and lethal enteric diseases and is a major animal welfare and economic problem for the poultry industry. Because repeated infectious episodes are possible, eradication is difficult in duck flocks. Despite advances in novel vaccines, Riemerellosis has mainly been controlled by chemotherapy. Quinolones, tetracyclines, and cephalosporins are widely used for controlling $R$. anatipestifer infection in the avian breeding industry, and consequently have led to the emergence of antibiotic-resistant strains. Recently, some classes of drug resistance genes have been identified in $R$. anatipestifer, including aminoglycoside resistance genes (ant, aac, and $a p h$ ), a chloramphenicol resistance gene (cat), and a chloramphenicol and florfenicol resistance gene (floR) [913].

Most studies of tigecycline focus on the bacteria found in humans, however, in many countries and areas, the variety of antibiotics used in the poultry industry is more than used in human medicine. In particular, in the avian breeding industry, the tigecycline analog tetracycline is commonly used and has resulted in increasing antibiotic drug resistance $[14,15]$. To date, tigecycline-resistance mechanisms in bacteria in the Flavobacteriaceae family have not been studied. In our previous study, we constructed a random transposon insertion library using the $R$. anatipestifer strain $\mathrm{CH} 3$ [16]. In this work, using the minimum inhibitory concentration (MIC) assay of tigecycline, we obtained a mutant strain which showed a significantly increased (about six-fold) tigecycline susceptibility compared with the wild-type strain $\mathrm{CH} 3$. To clarify whether the increased tigecycline susceptibility is associated with the knocked-down gene, the mutant strain and the inactivated gene were characterized. Our results indicate that $R$. anatipestifer M949_0459 gene is responsible for the bacterial resistance to tigecycline.

\section{RESULTS}

\section{Identification of the mutant showing increased tigecycline susceptibility}

In our previous work, about 2, 520 random transposon mutants were obtained [16], as mentioned above, using the tigecycline MIC assay, a mutant strain showed significantly increased (six-fold) tigecycline susceptibility (Table 2). With genome walking, the transposon-inserted gene was identified to be M949_0459, which encodes a putative flavin adenine dinucleotide (FAD)-dependent oxidoreductase of 386 amino acids, the mutant was named as $\mathrm{CH} 3 \Delta \mathrm{M} 949 \_0459$. The insertion of the transposon was located at nucleotide 112 of the gene (Figure 1). In addition, the effect of the M949_0459 gene deletion on expression of the genes in the flanking regions was investigated using qRT-PCR. As shown in Figure 2, the M949_0459 gene deletion inactivated the expression of the mutated gene, however, it did not affect the expression of both upstream gene M949_0460, which encodes the beta-lactamase class $\mathrm{D}$ protein, and the downstream gene M949_0458, which encodes a cation efflux protein, indicating transposon insertion in the M949_0459 gene had no polar effect on its flanking gene expression. Moreover, a stability test of the mutant strain CH3 $\triangle$ M949_0459 was performed based on the method of Fu and Tseng [20] with some modifications. The mutant strain CH3 $\triangle$ M949_0459 was subcultured in a non-selective medium for more than 50 generations. Approximately 200 colonies were selected, and each colony was patched into TSA plates supplemented with or without erythromycin. No difference in growth was observed between the two types of plates.

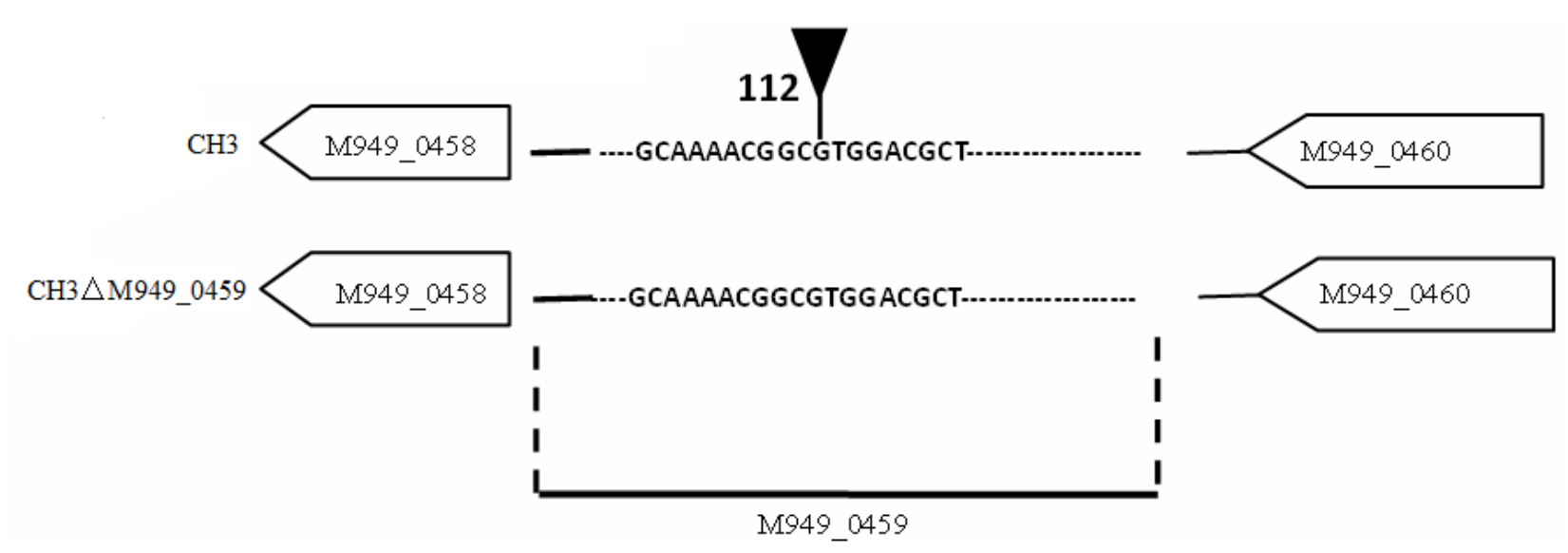

Figure 1: Schematic of the transposon insertion site. The tip of infundibulum indicates the transposon insertion site (112 bp). 
Table 1: Strains, plasmids, and primers used in this study

\begin{tabular}{|c|c|c|}
\hline Strains, plasmids or primers & Description & References \\
\hline \multicolumn{3}{|l|}{ Strains } \\
\hline $\mathrm{CH} 3$ & Riemerella anatipestifer wild-type strain (serotype 1) & {$[49]$} \\
\hline CH3 $\Delta$ M949_0459 & $\begin{array}{l}\text { Mutant strain, derived from Riemerella anatipestifer } \mathrm{CH} 3 \text {, in which the } \\
\text { M949 } 0459 \text { gene was inactivated by Tn } 4351 \text { insertion. }\end{array}$ & This study \\
\hline \multicolumn{3}{|l|}{ plasmids } \\
\hline Pet-28a $(+)$ & $\operatorname{Kan}^{\mathrm{r}}$, Bacterial expression vector with T7 lac promoter & {$[50]$} \\
\hline Pet-28a(+)-M949_0459 & $\begin{array}{l}\mathrm{Kan}^{\mathrm{r}} \text {, Bacterial expression vector with } \mathrm{T} 7 \text { lac promoter and the } \\
\text { M949_0459 gene insertion }\end{array}$ & This study \\
\hline \multicolumn{3}{|c|}{ 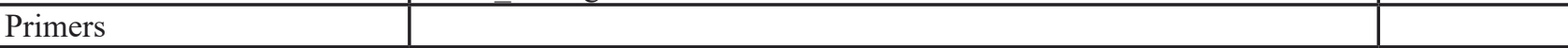 } \\
\hline RA 16S rRNA & 5'-GAGCGGTAGAGTATCTTCGGATACT-3' & This study \\
\hline RA 16S rRNA & 5'-AATTCCTTTGAGTTTCAACCTTGCG-3' & This study \\
\hline SP1 & 5'-CTCCCAGAAAATTTCCAAGACTCTCA-3' & This study \\
\hline SP2 & 5'-TAAAGTGCTGACCCGTAAAACGAAC-3' & This study \\
\hline SP3 & 5'-GTGGTAGCTATAGCATGGAGCTTGC-3' & This study \\
\hline RTPCR-16SF & 5'-CAACCATGCAGCACCTTGAAAA -3' & This study \\
\hline RTPCR-16SR & 5'-GACGAAAGCGTGGGGAGCGAAC-3' & This study \\
\hline M949_0459-F & 5'-ACAAAGACCGAGATGCCAGG -3' & This study \\
\hline M949_0459-R & \begin{tabular}{|l} 
5'-GTCAAACCGATTTTCGGGCT -3' \\
\end{tabular} & This study \\
\hline M949_0458-F & 5'-GCTGTTGGCGGAACAGTAGT-3' & This study \\
\hline M949_0458-R & 5'-GTCAGGCATTTTCTCGACTCC-3' & This study \\
\hline M949_0460 -F & 5'-AGGAATGGTTTTCGATACTTTGACG-3' & This study \\
\hline M949_0460-R & 5'-CCAGCCTGATTTCCCGTTCA-3' & This study \\
\hline
\end{tabular}

\section{Homologous gene and protein analyses.}

Currently, 28 complete $R$. anatipestifer genomes have been submitted to NCBI, BLAST analysis indicated that the M949_0459 homologous sequence was observed in 6 of them (21\%), suggesting that the distribution of the homologous sequence is limited in partial Riemerella strains. By BLASTP and FASTA algorithms, the M949_0459 presents 100\% identity with G148_RS08775 and G148_RS08830 (RA-CH-2), 99\% identity with B739_RS00155 (RA-CH-1), 95\% identity with B739_

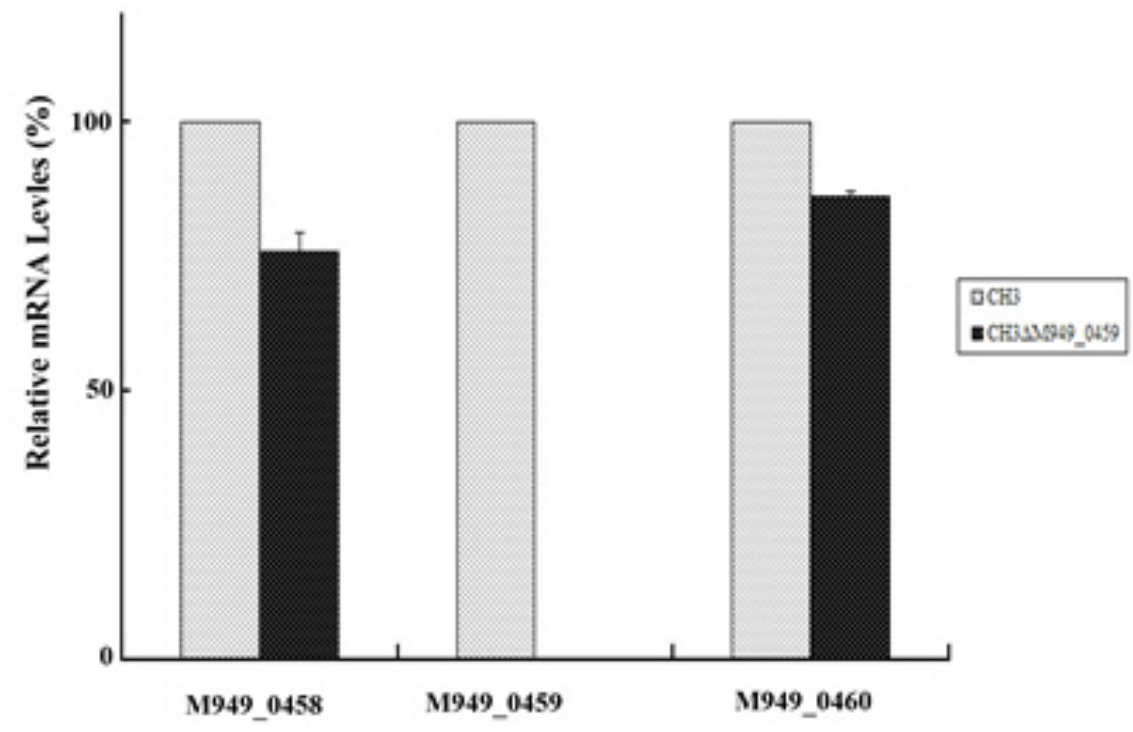

Figure 2: The M949_0459 gene deletion did not affect expression of its flanking genes. The expression of the M949_0459 and its flanking genes in the mutant strain CH3 $\triangle$ M949_0459 and wild-type strain CH3 was determined using quantitative real-time PCR analysis. The expression of M949_0459 gene was inactivated in the mutant strain CH3 $\triangle$ M949_0459, however, no significant difference was shown in expression of M949_0458 and M949_0460 genes between R. anatipestifer CH3 and CH3 $\triangle$ M949_0459. Error bars represent standard deviations from three replicates. 
Table 2: Determination of bacterial susceptibility to antibiotics

\begin{tabular}{|c|c|c|c|c|c|c|c|c|}
\hline \multirow[b]{2}{*}{ Strains } & \multicolumn{8}{|c|}{ Antibiotics } \\
\hline & 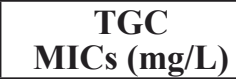 & AMP & NOR & CHL & CRO & ERY & GEN & KAN \\
\hline $\mathrm{CH} 3$ & 12.0 & $\mathrm{~S}$ & $\bar{I}$ & $\mathrm{R}$ & $\bar{S}$ & $\mathrm{~S}$ & $\mathrm{R}$ & $\mathrm{R}$ \\
\hline CH3 $\triangle$ M949 0459 & 2.0 & $\mathrm{~S}$ & I & $\mathrm{R}$ & $\mathrm{S}$ & $\mathrm{R}$ & $\mathrm{R}$ & $\mathrm{R}$ \\
\hline
\end{tabular}

Abbreviations: TGC, tigecycline; AMP, ampicillin; NOR, norfloxacin; CHL, chloromycetin; CRO, ceftriaxone; ERY, erythromycin; GEN, gentamicin; KAN, kanamycin. MIC, the minimum inhibitory concentration; S, susceptible; I, intermediate; $\mathrm{R}$, resistant.

RS00130 (RA-CH-1), 94\% identity with RIA_RS01715 (RA-GD) and AS87_09615 (Yb2), and 93\% identity with RIA_RS01735 (RA-GD). Multi-sequence alignment of the $\bar{M} 949$ 0459 homologous genes from different sources and evolutionary relation of the M949_0459 homologous proteins from different sources were summarized in Figure 3. Furthermore, comparative analyses of the M949_0459 gene environment indicated that the upstream M949_0460 homologous gene was observed in 4 of the 5 genomes, and the downstream M949_0458 homologous gene was observed in 2 of the 5 genomes (Figure 4).

\section{Knock-down of the M949_0459 gene decreased bacterial metabolic activity}

FAD-dependent oxidoreductase is a complex I $\mathrm{NADH}$ ubiquinone oxidoreductase that is crucial for transferring electrons into the respiratory chain in bacteria [21]. Mutations in FAD-dependent oxidoreductase may result in metabolic deficiencies. Based on the annotation of the M949_0459 as a putative FAD-dependent oxidoreductase in the GenBank, we tested the viability and metabolism of the bacteria using the alamarBlue assay. The results showed that the metabolic activity clearly decreased in the mutant strain CH3 AM949_0459 compared with the wild-type strain $\mathrm{CH} 3$ (Figure 5).

\section{The M949_0459 gene conferred tigecycline resistance to transformed $E$. coli BL21}

We measured the uptake of tigecycline by $E$. coli BL21 (DE3) which contained M949 0459 on a Pet28a(+) background. The results showed that M949_0459 conferred tigecycline resistance to E. coli BL21 (Table 3 , Figure 6A) but did not affect susceptibility to other antibiotics including ampicillin, norfloxacin, ceftriaxone, gentamicin, kanamycin and imipenem. As mentioned above, sequence analysis of M949_0459 revealed that this gene shared 91\% similarity to TetX (Bacteroides fragilis str. S23L17). In 1988, Speer and Salyers first identified the Tet $X$ gene from a Bacteroides fragilis strain [22]. Further research discovered that TetX is a flavin-dependent monooxygenase that adds a hydroxyl group to the antibiotic to inactive tetracycline, and more importantly, with the presence of tetracycline, introduction of the tet $X$ gene into $E$. coli induced a color change from orange to brown in the growth media [23]. Additionally, some evidence has shown that tigecycline and tetracycline act in a similar manner by binding to the same site on the ribosome [24, 25], our studies have indicated that an oxygen-dependent color change was observed in the presence of tigecycline (Figure 6B).

Tigecycline treatment changed expression of the M949_0459 gene in the bacteria at transcriptional level

By qRT-PCR, we quantitatively examined the expression of M949 0459 following treatment with different doses of tigecycline. We observed a non-linear effect of the tigecycline dose on the M949_0459 gene expression. The results showed that the mRNA expression of the M949_0459 gene was elevated under tigecycline treatment with dose range of $1-10 \mathrm{mg} / \mathrm{L}$, and peaked at 4 mg/L (Figure 7).

\section{The efflux pump inhibitors (EPIs) played no roles on the tigecycline susceptibility}

In contrast to destruction or covalent modification of other antibiotics such as $\beta$-lactams and aminoglycosides, efflux systems have been identified as the primary mechanism of tigecycline resistance in Klebsiella pneumonia, Proteus mirabilis, and E. coli [26-28]. In this study, two kinds of EPIs, PA $\beta \mathrm{N}$ and CCCP, were used to evaluate the role of the efflux pumps in tigecycline resistance in $R$. anatipestifer strain $\mathrm{CH} 3$. As shown in Table 4, the tigecycline susceptibility of $R$. anatipestifer strain $\mathrm{CH} 3$ and $\mathrm{CH} 3 \Delta \mathrm{M} 949$ 0459 showed no difference with or without the EPIs, indicating the efflux pump inhibitors (EPIs) play no roles on the tigecycline susceptibility.

\section{DISCUSSION}

As a glycylcycline antimicrobial agent, tigecycline has been used for therapy in humans against a wide range of Gram-positive and Gram-negative anaerobic 
A

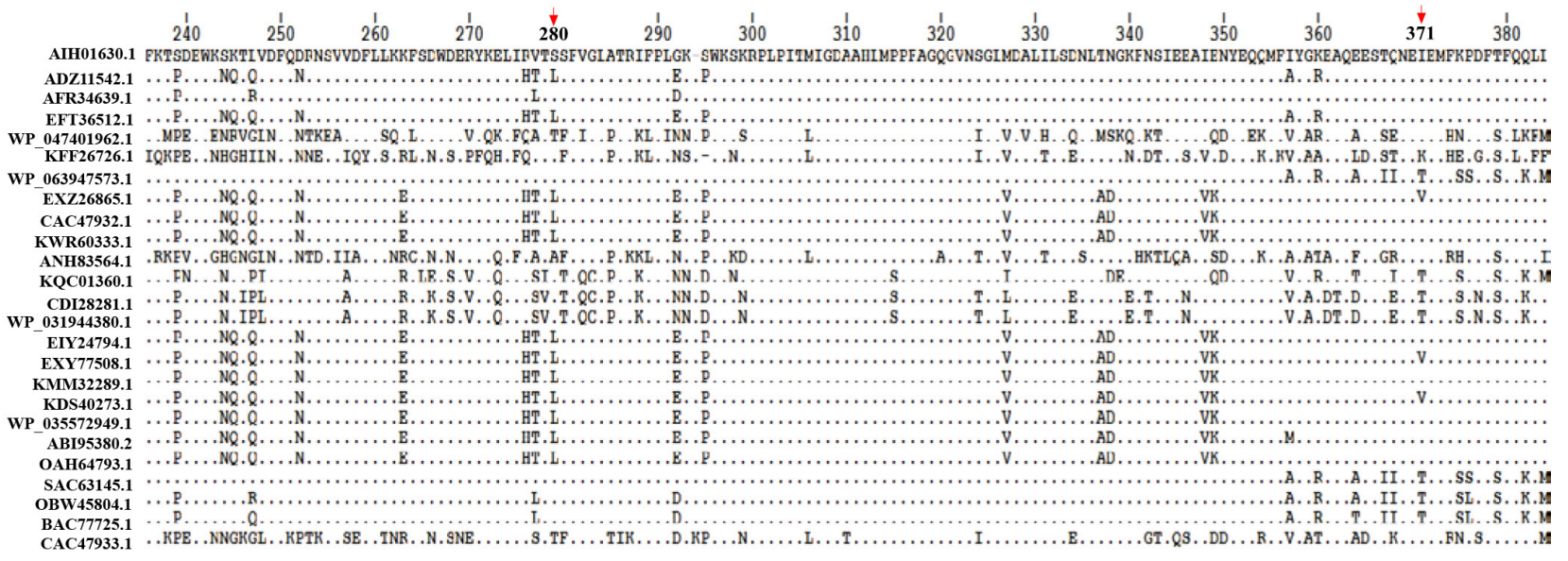

B

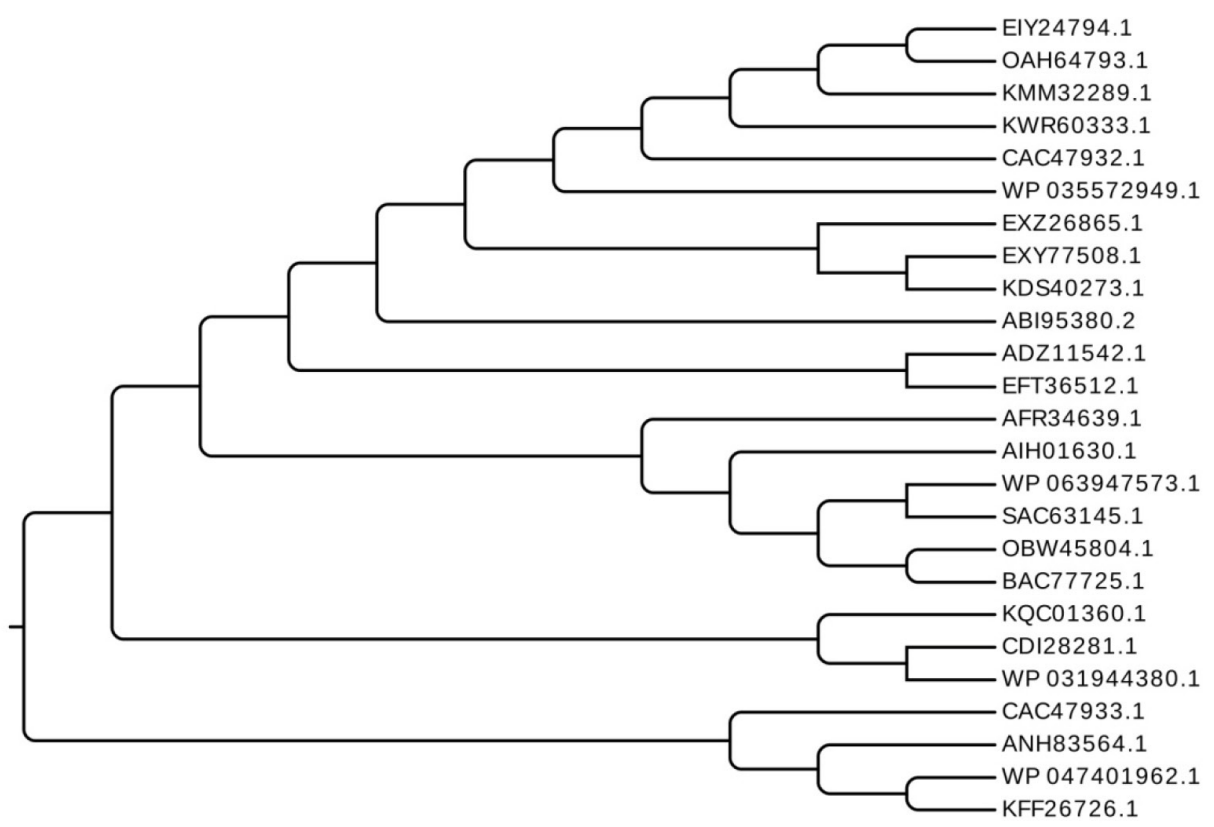

Figure 3: Bioinformatics analysis. A. Multi-sequence alignment of the M949_0459 homologous proteins from different sources. The arrows indicate the locations of 280th and 371th amino acids. B. Evolutionary relation of the M949_0459 homologous proteins from different sources. Twenty five representatives were selected for the multi-sequence alignment and evolutionary relation analysis, including AIH01630.1 FAD-dependent oxidoreductase ( $R$. anatipestifer CH3), ADZ11542.1 FAD-dependent oxidoreductase ( $R$. anatipestifer RAGD), AFR34639.1 hypothetical protein B739_0030 (R. anatipestifer RA-CH-1), EFT36512.1 monooxygenase, FAD-binding protein (R. anatipestifer RA-YM), WP_047401962.1 tetracycline resistance protein (Chryseobacterium sp. YR460), KFF26726.1 tetracycline resistance protein (Chryseobacterium piperi), WP 063947573.1 TetX family tetracycline inactivation enzyme (Enterobacter cloacae), EXZ26865.1 tetX2 protein (Bacteroides fragilis str. S36L11), CAC47932.1 TetX2 protein (Bacteroides thetaiotaomicron), KWR60333.1 kynurenine 3-monooxygenase (Bacteroides ovatus), ANH83564.1 tetracycline resistance protein (Niabella ginsenosidivorans), KQC01360.1 tetracycline resistance protein (Pedobacter sp. Hv1), CDI28281.1 FAD dependent oxidoreductase (Acinetobacter pittii 42F), WP_031944380.1 TetX family tetracycline inactivation enzyme (Acinetobacter calcoaceticus), EIY24794.1 hypothetical protein HMPREF1064_05159 (Bacteroides dorei CL02T12C06), EXY77508.1 tetX2 protein (Bacteroides fragilis str. 3988 T1), KMM32289.1 tetracycline resistance protein (Parabacteroides goldsteinii), KDS40273.1 tetX2 protein (Bacteroides vulgatus str. 3775 SRB19), WP_035572949.1TetX family tetracycline inactivation enzyme (Elizabeth kingiaanophelis), ABI95380.2 tetracycline resistance protein (Sphingobacterium sp. PM2-P1-29), OAH64793.1 tetracycline resistance protein (Chryseobacterium sp. J200), SAC63145.1 Kynurenine 3-monooxygenase (Enterobacter cloacae), OBW45804.1 Kynurenine 3-monooxygenase (Chryseobacterium sp. BGARF1), BAC77725.1 tetracycline inactivating enzyme (Pseudomonas aeruginosa), and CAC47933.1 TetX1 protein (Bacteroides thetaiotaomicron), respectively. The multi-sequence alignments were performed using CLUSTAL Omega. 
Table 3: Determination of the tigecycline susceptibility of $E$. coli BL21 transformed with Pet-28a (+)-M949_0459

\begin{tabular}{|l|l|l|l|l|l|l|l|}
\hline \multirow{2}{*}{\multicolumn{1}{c|}{ Strains }} & \multicolumn{6}{c|}{ Antibiotics } \\
\cline { 2 - 8 } & $\begin{array}{c}\text { TGC } \\
\text { MICs (mg/L) }\end{array}$ & \multicolumn{1}{|c|}{ AMP } & NOR & CRO & GEN & KAN & IPM \\
\hline Pet28a(+)-DE3 & 0.25 & S & S & S & S & R & S \\
\hline Pet-28a(+)-M949_0459 & 1.00 & S & S & S & S & R & S \\
\hline
\end{tabular}

Abbreviations: TGC, tigecycline; AMP, ampicillin; NOR, norfloxacin; CRO, ceftriaxone; GEN, gentamicin; KAN, kanamycin; IPM, imipenem; MIC, the minimum inhibitory concentration; S, susceptible; R, resistant.

and aerobic bacteria and cell-wall free mycoplasmas, chlamydiae, and mycobacterium [29]. Although there have been no reports on the use of tigecycline against bacteria unique to a food animal, however, the first identified bacteria carrying special plasmids and antibiotic resistance genes were found in humans, which could make their way into food animal bacteria and vice versa. In many countries and areas, the total amount of antibiotics used in the poultry industry is more than the amount used in human medicine and is thus an important element in exposing bacteria to antibiotics [30-32]. In particular, in the avian breeding industry, the tigecycline analog tetracycline is commonly used and has resulted in increasing antibiotic drug resistance [33]. At present, increasing numbers of reports on tigecycline-resistant bacterial strains isolated from human sources are of concern, yet the molecular mechanisms regarding tigecycline resistance are poorly understood.

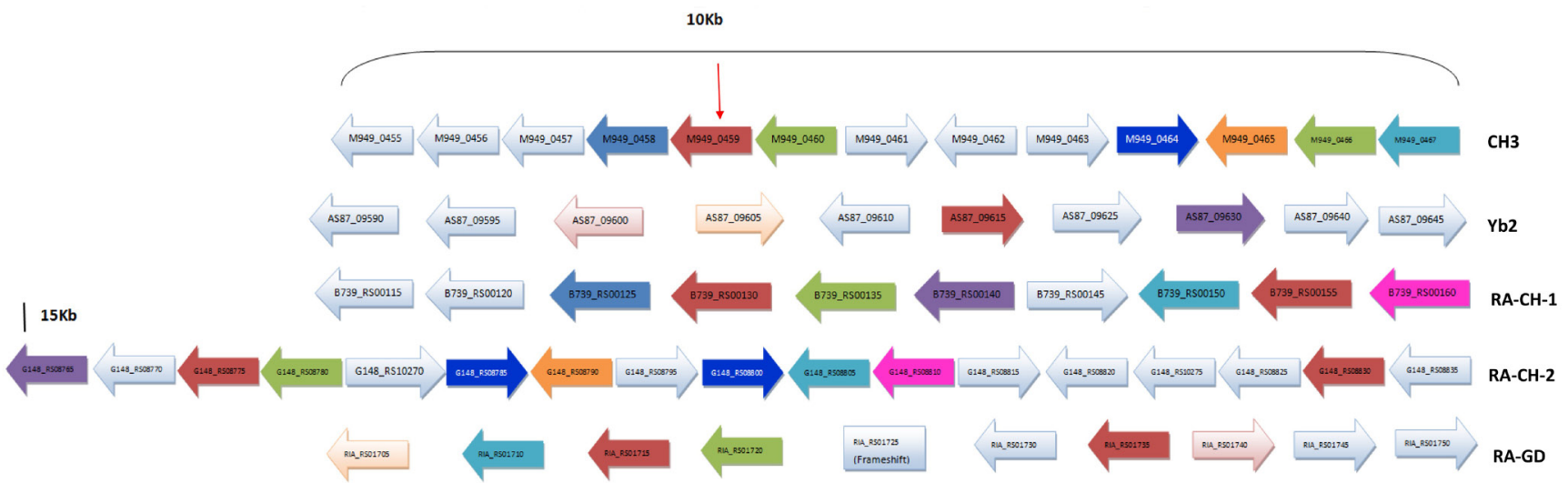

Figure 4: Gene environment around the M949_0459 and its homology genes in Riemerella genomes. The arrow indicates the M949_0459 gene. The same color indicates the homology gene.

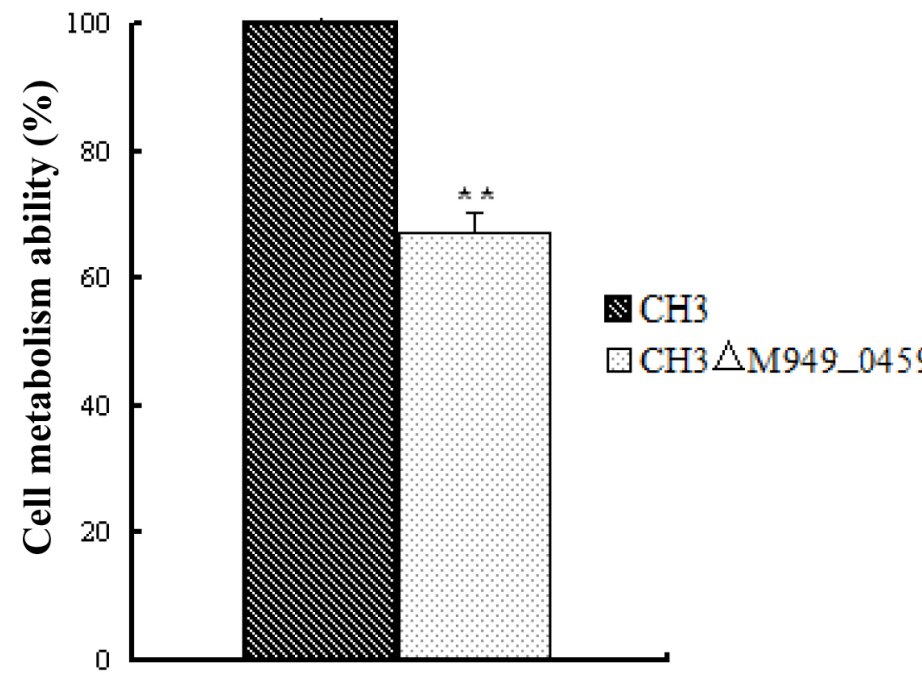

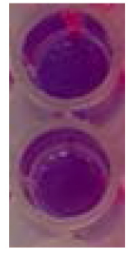

LB medium
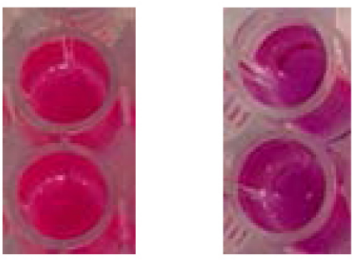

CH3

CH3 $\triangle$ M949_0459

Figure 5: Knock-down of the M949_0459 gene decreased bacterial metabolic activity. The metabolic activity of the strains was measured using the alamarBlue reagent. ${ }^{*} p<0.01$. 
Table 4: Effects of efflux pump inhibitors on tigecycline susceptibility

\begin{tabular}{|l|l|l|}
\hline \multirow{2}{*}{ Strains } & \multicolumn{2}{c|}{ TGC MICs (mg/L) } \\
\cline { 2 - 4 } & \multicolumn{1}{|c|}{ Without EPIs } & \multicolumn{1}{c|}{ With inhibition of EPIs } \\
\hline $\mathrm{CH} 3$ & $12.0 \quad 2.0$ \\
\hline $\mathrm{CH} 3 \Delta$ M949_0459 & 2.0 & 2.0 \\
\hline
\end{tabular}

Abbreviations: TGC, tigecycline; MIC, the minimum inhibitory concentration; EPIs, efflux pump inhibitors, including carbonyl cyanide $\mathrm{m}$-chlorophenyl hydrazone $(3 \mathrm{mg} / \mathrm{L})$ and phenylalanine arginyl $\beta$-naphthylamide $(40 \mathrm{mg} / \mathrm{L})$.

$R$. anatipestifer is ubiquitous in the environment, and has been isolated from ducks, turkeys, chickens, and other birds $[9,34]$. In farmed ducks, $R$. anatipestifer infection leads to high mortality and consequently to great economic losses. Currently, a variety of approaches have been applied to control $R$. anatipestifer infections, but the most widely used strategy is chemotherapeutic agents in commercial poultry flocks, despite rising drug resistance. To date, several antibiotic resistance genes have been reported in $R$. anatipestifer $[10,11]$. Using the tigecycline MIC assay, the M949_0459 gene-deficient $R$. anatipestifer mutant strain CH3 $\triangle \mathrm{M} 9490459$ had significantly increased tigecycline susceptibility compared with the wild-type strain $\mathrm{CH} 3$. Sequencing analysis revealed that

A

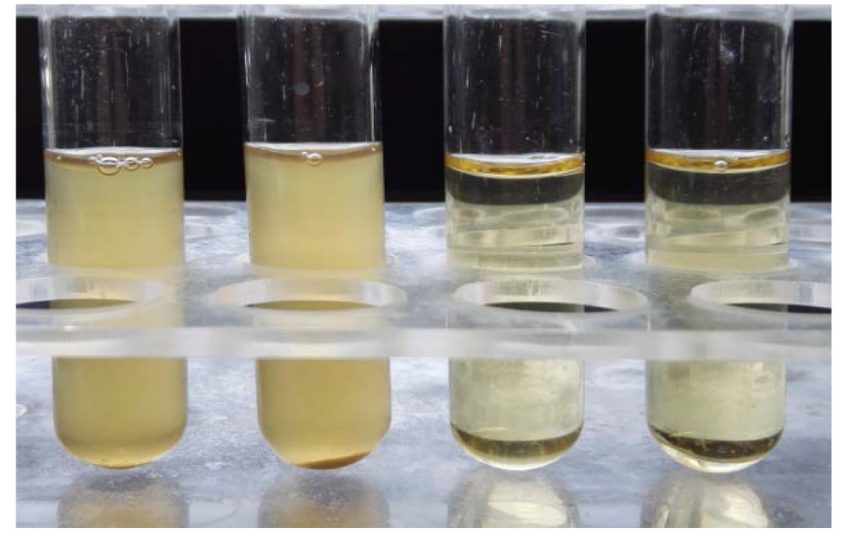

FAD-Pet28a(+)-M949_0459

Pet28a (+)-DE3

(0.5 mg/L TGC)

$(0.5 \mathrm{mg} / \mathrm{L}$ TGC)

B

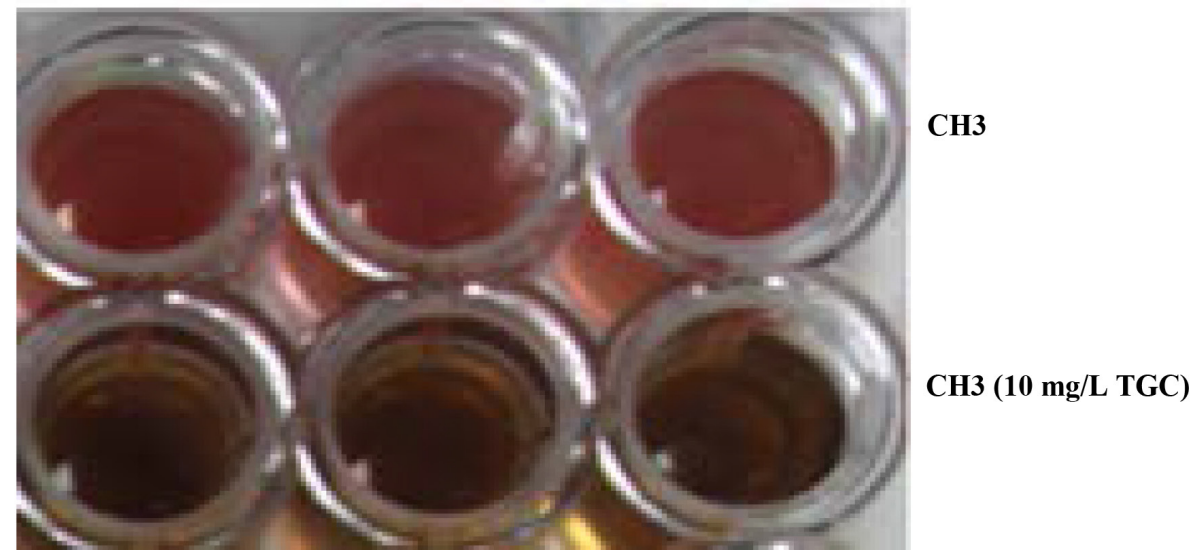

Figure 6: Determination of the tigecycline susceptibility of $E$. coli BL21 transformed with Pet-28a(+)-M949_0459. A. The transformants containing the M949_0459 gene showed higher tigecycline resistance compared to controls. B. An oxygen-dependent color change was observed in the presence of high doses of tigecycline. 
the M949_0459 gene encodes a putative FAD-dependent oxidoreductase. To confirm that the M9490459 is the resistance gene for tigecycline, M949_0459 on a Pet28a(+) background was transformed into $\bar{E}$. coli BL21 (DE3). As we had hypothesized, the expression of the recombinant protein conferred tigecycline resistance to $E$. coli BL21 (DE3) cells. Previous studies about the FADdependent oxidoreductase related to the resistance to the tigecycline analog tetracycline had demonstrated that the resistance mechanism of tetracycline was identified with detoxification of tetracycline, and resulted in a change in the color of the medium from yellow to gray-black [35]. In our current work, we showed a similar phenomenon in the E. coli BL21 (DE3) expressing M949_0459.

Although it is known that FAD-dependent oxidoreductase can alter tigecycline to 11a-hydroxytigecycline, resulting in a weakened ability to block protein translation [36], there have been no studies that demonstrate differences in FADdependent oxidoreductase protein expression induced by various doses of tigecycline. In our present work, the dose-response relationship between tigecycline and the mRNA level of M949 0459 was measured. In the absence of tigecycline, there was detectable expression of M949_0459. At a $4 \mathrm{mg} / \mathrm{L}$ dose, there was a significant increase in expression, followed by a slight increase at 2 $\mathrm{mg} / \mathrm{L}$. However, when the dose of tigecycline increased from $4 \mathrm{mg} / \mathrm{L}$ to $10 \mathrm{mg} / \mathrm{L}$, on the contrary, the M949_0459 gene expression was gradually decreased, and finally was expressed at an extremely low level.
In addition to FAD-dependent oxidoreductase, resistance to tigecycline in the clinic also occurs through an efflux protein-mediated mechanism in bacteria. Efflux pumps can transport a specific substrate or a range of structurally dissimilar compounds. The latter efflux pumps are related to multidrug (antibiotic) resistance (MDR) [37, 38]. In bacteria, five families of efflux pump proteins have been described: the ATP-binding cassette superfamily, the major facilitator superfamily (MFS), the multidrug and toxic-compound extrusion (MATE) family, the small multidrug resistance (SMR) family, and the RND family [39]. Previous studies have shown that reduced tigecycline susceptibility mainly results from the overexpression of the RND pumps, AcrAB-TolC, and MATE pumps in different bacteria [40]. This led us to assess efflux pumps as an alternative tigecycline resistance mechanism in the current study. In our present work, two broad-spectrum EPIs, CCCP and PA $\beta \mathrm{N}$, were used to identify the phenotype of active efflux systems. The results indicated that these two EPIs did not affect the susceptibility to tigecycline. Li et al. described the effect of $\mathrm{CCCP}$ and PA $\beta \mathrm{N}$ on the MIC of ten antibiotic agents against 66 strains of $R$. anatipestifer clinic isolates. They found that these two EPIs restored susceptibility of $R$. anatipestifer isolates to tetracycline [41]. Nevertheless, they did not examine the effect of these two EPIs on the susceptibility of $R$. anatipestifer isolates to tigecycline. It is possible that, different from tetracycline, tigecycline has a lower affinity for specific efflux pumps [42]. It is also possible that tigecycline cannot be expelled by MFS efflux

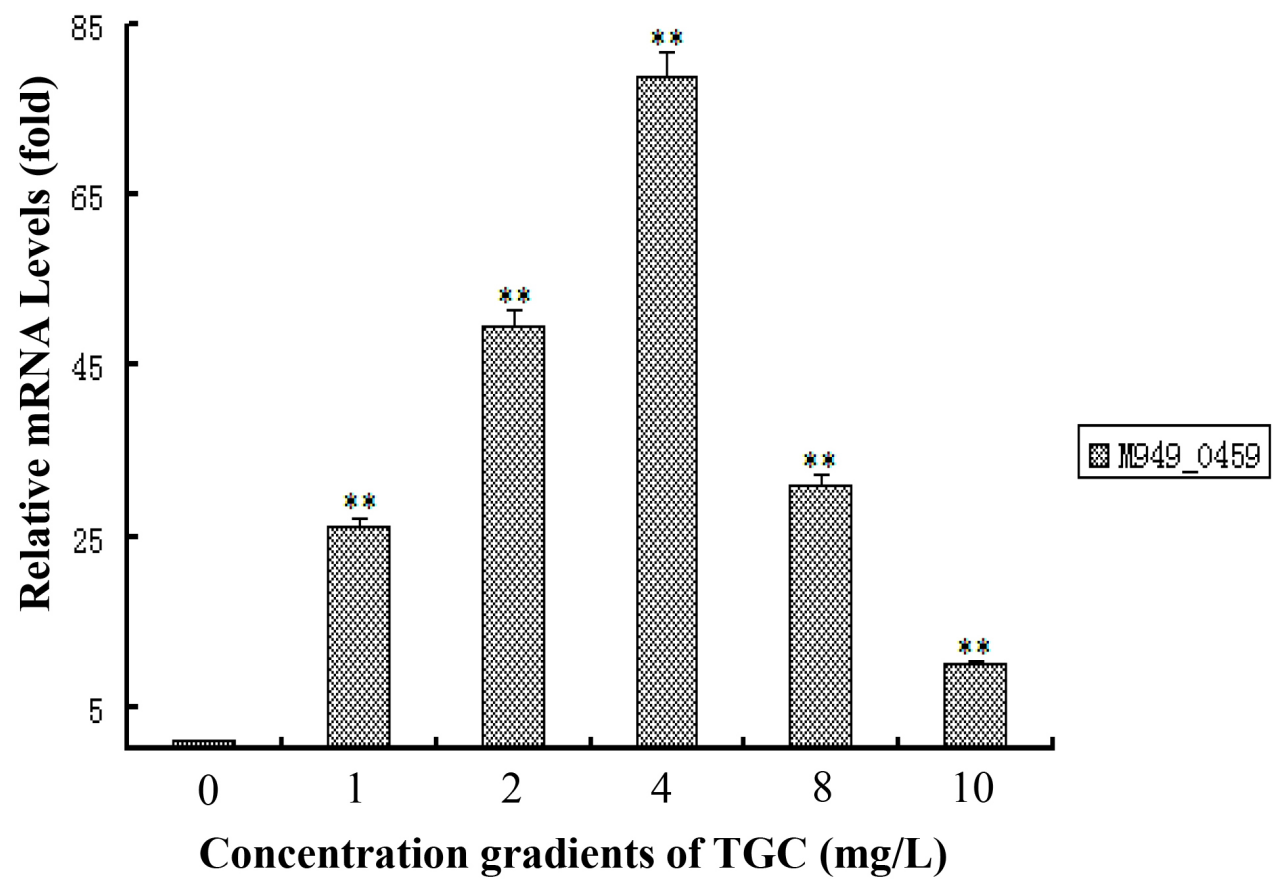

Figure 7: Tigecycline treatment changed the expression of M949_0459 gene. The expression of the M949_0459 gene following treatment with different concentrations of tigecycline was measured by quantitative real-time PCR. Error bars represent standard deviations from three replicates. ${ }^{* *} p<0.01$. 
pumps in either Gram-negative or Gram-positive bacteria [42]. Alternatively, it may be that different kinds of EPIs interfere with efflux pump activity through different modes of action [43]. Currently, no reports about MDR pumps in $R$. anatipestifer have been published, although genome analysis data has indicated that hypothetical MDR efflux pumps, such as those belonging to the MFS and SMR families, exist in $R$. anatipestifer [10, 44, 45]. Further research seeking specific resistance pumps playing important roles in extruding antimicrobial agents in $R$. anatipestifer is needed.

It is intriguing that the amino acid sequence deduced by M949_0459 shares 91\% sequence identity with the TetX2 protein (B. fragilis str. S23L17). Yang et al. first demonstrated that TetX2 belongs to a family of FAD-requiring monooxygenases that inactivate a broad selection of tetracycline antibiotics through reductive electron transfer or hydroxylation reactions [46]. Recently, Walkiewicz evaluated the potential of TetX tetracycline resistance proteins to acquire mutations causing tigecycline resistance. They found that among TetX mutants showing higher resistance levels to tigecycline, more than half contained the amino acid substitution as a single mutation or in combination with other changes at position 280. Furthermore, assessing the fitness of $E$. coli containing one copy of either wild-type TetX2 or variants of TetX2 amino acid substitution with a range of minocycline concentrations, the amino acid substitution T280A/T280S, and N371I/N371T were succeed in the bacterial drug adaptation experiment $[47,48]$. In this study, a comparison of the sequences from M949_0459 and the other selected TetX sequences showed that the spontaneous amino acid substitution is present in M949_0459 at position 280 and 371 (Figure 3A).

In summary, we identified that $R$. anatipestifer M949_0459 gene is responsible for the bacterial resistance to tigecycline, which may provide insights into molecular mechanisms for chemotherapy in combating $R$. anatipestifer infections.

\section{MATERIALS AND METHODS}

\section{Bacterial strains, plasmids, and media}

The bacterial strains and plasmids used in this study are listed in Table 1. R. anatipestifer wild-type strain CH3 and the mutant strain CH3 $\triangle$ M949_0459 were used. The mutant strain CH3 $\mathrm{CM} 949 \_0459$ was screened out from a random transposon mutant library that was previously constructed in our laboratory [16] using the tigecycline MIC assay. $R$. anatipestifer strains were grown in broth microdilutions according to the Clinical and Laboratory Standards Institute (CLSI) at $37^{\circ} \mathrm{C}$. E. coli strains were grown at $37^{\circ} \mathrm{C}$ on Luria-Bertani (LB) plates or in LB broth. When necessary, the medium was supplemented with appropriate antibiotics at the following concentrations: ampicillin (100 mg/L), chloramphenicol (30 $\mathrm{mg} / \mathrm{L})$, erythromycin $(0.5 \mathrm{mg} / \mathrm{L})$, kanamycin $(50$ $\mathrm{mg} / \mathrm{L})$, and cefoxitin $(5 \mathrm{mg} / \mathrm{L})$.

\section{Antimicrobial susceptibility test}

The MIC value of tigecycline was determined by serial dilution in 96-well culture plates, with these being performed according to the guide published by the CLSI in 2015. Other antimicrobial drug susceptibility tests were tested by the Kirby Bauer disc diffusion method according to CLSI guidelines in 2015.

\section{Identification of the mutant strain}

The transposon insertion site in the mutant strain was determined with genome walking, which was performed with a genome walking kit (TaKaRa, Dalian, China) using four random primers (AP1, AP2, AP3, and AP4) provided in the kit and three specific primers (SP1, SP2, and SP3), according to the manufacturer's instructions. The PCR-amplified gene was inserted into T-vector pMD19 (TaKaRa) for DNA sequencing (Shanghai HuaGene Biotech, Shanghai, China). The identified gene sequence was searched for homologous sequences on the BLAST server (http://blast.st-va.ncbi.nlm.nih.gov/Blast.cgi).

\section{Bioinformatics analysis}

The COGnitor software (http://www.ncbi.nlm.nih. gov/COG/old/xognitor.html) was used to assess putative functions of the gene. The homologous protein sequences were searched using the BLASTX server (http://www. ncbi.nlm.nih.gov/BLASTX/) and aligned using CLUSTAL Omega [17].

\section{Quantitative real-time PCR analysis}

Quantitative real-time PCR (qRT-PCR) was performed to measure the expression levels of the transposon-disrupted and flanking genes in wild-type strain CH3 and mutant strain, as well as the M949_0459 gene following treatment with different doses of tigecycline at the transcriptional level. Total RNA of the samples was extracted with TRIzol reagent (Invitrogen, Carlsbad, CA, USA) according to the manufacturer's instructions. To avoid DNA contamination, the extracted RNA was treated with RNase-free DNase I (40 U/mg RNA, TaKaRa) and purified using the RNeasy Mini Kit (Qiagen, Hilden, Germany) according to the manufacturer's instructions. cDNA was synthesized using the GoTaq qPCR Master Mix Kit (Promega, Madison, WI, USA) following the 
manufacturer's instructions after adjusting the samples to the same concentration. qRT-PCR was performed with the ABI7500 Real-Time PCR System (Applied Biosystems, Foster City, CA, USA). Gene-specific primers were designed using the primer3 online software v.0.4.0 (http:// bioinfo.ut.ee/primer3-0.4.0/) and are described in Table 1. The expression of $16 \mathrm{~S}$ rRNA was used as an internal control. The specificity of amplification was confirmed using melting curve and electrophoresis analysis. The data were analyzed with the normalized gene expression method (2- $\Delta \Delta \mathrm{CT})$ [18]. Each reaction was performed in triplicate, and the entire experiment was carried out in triplicate.

\section{alamarBlue assay}

The metabolic activity of the strains was measured using the alamarBlue reagent (Thermo Fisher, Waltham, MA, USA), according to the manufacturer's protocol. In brief, $20 \mu \mathrm{L}$ of the alamarBlue reagent was directly added to $200 \mu \mathrm{L}$ of the bacterial culture medium and blank medium (negative control); then, these were incubated for $1 \mathrm{~h}$ at $37^{\circ} \mathrm{C}$ while protected from direct light. Next, the absorbance of alamarBlue was monitored at $570 \mathrm{~nm}$, using $600 \mathrm{~nm}$ as a reference wavelength (normalized to the $600 \mathrm{~nm}$ value). The proliferation rate $(\mathrm{r})$ was calculated according to the following formula: $\left[117,216 \times \mathrm{A}_{570}\right.$ (sample) - 80, 856 $\times \mathrm{A}_{600}$ (sample) $] /\left[117,216 \times \mathrm{A}_{570}\right.$ (control) $-80,856 \times \mathrm{A}_{600}($ control $\left.)\right] \times 100 \%$.

\section{Construction and transformation of the Pet- 28a(+)-M949_0459 plasmid}

The DNA fragment carrying the M949_0459 gene was amplified from the $R$. anatipestifer strain $\mathrm{CH} 3$ using the primers listed in Table 1. After amplification, the amplimer was cloned into the expression vector Pet-28a(+) to construct Pet-28a(+)-M949_0459 plasmid, and E. coli strain BL21 was subsequently used for transformation. Using Luria-Bertani agar (Difco, Detroit, MI, USA) containing kanamycin $(50 \mathrm{mg} / \mathrm{L})$, the transformants were selected and sequenced. The confirmed transformants were subjected to tigecycline susceptibility testing. E. coli BL21 with Pet-28a(+) vector without the DNA insertion was used as a control.

\section{Efflux pump inhibition assay}

An efflux pump inhibition assay was performed as described previously [19]: broth microdilution testing of tigecycline was performed with and without phenylalanine arginine $\beta$-naphthylamide $(\mathrm{PA} \beta \mathrm{N})(40 \mathrm{mg} / \mathrm{L})$ and carbonyl cyanide m-chlorophenyl hydrazone (CCCP) $(3 \mathrm{mg} / \mathrm{L})$.

\section{Statistical analysis}

Statistical analysis was performed with Student's $t$ test. Mean values are shown in the figures. Statistical significance was established at $p<0.05$.

\section{Author contributions}

TL and SY planned the experiments. TL, MS and JH conducted the experiments. TL, XW, SW, MT, JQ, YS, $\mathrm{TL}, \mathrm{CD}$, and SY analyzed and discussed the experimental results. TL and SY wrote the manuscript.

\section{CONFLICTS OF INTEREST}

The authors declare that the research was conducted in the absence of any commercial or financial relationships that could be construed as a potential conflict of interest.

\section{FUNDING}

This work was supported by the National Key R \& D Program (2016YFD0500805), the Shanghai Key Project on Agricultural Development through Science and Technology (2016HNG4-1) and Co-innovation of Science and Technology Innovation Project in Chinese Academy of Agricultural Sciences (CAAS-XTCX2016011-04-8).

\section{REFERENCES}

1. Petersen PJ, Jacobus NV, Weiss WJ, Sum PE, Testa RT. In vitro and in vivo antibacterial activities of a novel glycylcycline, the 9-t-butylglycylamido derivative of minocycline (GAR-936). Antimicrob Agents Chemother. 1999; 43: 738-744.

2. Sum PE, Petersen P. Synthesis and structure-activity relationship of novel glycylcycline derivatives leading to the discovery of GAR-936. Bioorg Med Chem Lett. 1999; 9: 1459-1462.

3. French GL. A review of tigecycline. J Chemother. 2008; 20: 3-11.

4. Nowak J, Seifert H, Higgins PG. Prevalence of eight resistance-nodulation-division efflux pump genes in epidemiologically characterized Acinetobacter baumannii of worldwide origin. J Med Microbiol. 2015; 64: 630-635.

5. McQueary CN, Kirkup BC, Si Y, Barlow M, Actis LA, Craft DW, Zurawski DV. Extracellular stress and lipopolysaccharide modulate Acinetobacter baumannii surface-associated motility. J Microbiol. 2012; 50: 434-443.

6. Worthington RJ, Richards JJ, Melander C. Small molecule control of bacterial biofilms. Org Biomol Chem. 2012; 10: 7457-7474.

7. Li X, Liu L, Ji J, Chen Q, Hua X, Jiang Y, Feng Y, Yu 
Y. Tigecycline resistance in Acinetobacter baumannii mediated by frameshift mutation in plsC, encoding 1-acylsn-glycerol-3-phosphate acyltransferase. Eur J Clin Microbiol Infect Dis. 2015; 34: 625-631.

8. Segers P, Mannheim W, Vancanneyt M, De Brandt K, Hinz $\mathrm{KH}$, Kersters K, Vandamme P. Riemerella anatipestifer gen. nov., comb. nov., the causative agent of septicemia anserum exsudativa, and its phylogenetic affiliation within the Flavobacterium-Cytophaga rRNA homology group. Int J Syst Bacteriol. 1993; 43: 768-776.

9. Sun N, Liu JH, Yang F, Lin DC, Li GH, Chen ZL, Zeng ZL. Molecular characterization of the antimicrobial resistance of Riemerella anatipestifer isolated from ducks. Vet Microbiol. 2012; 158: 376-383.

10. Luo H, Liu M, Wang L, Zhou W, Wang M, Cheng A, Jia R, Chen S, Sun K, Yang Q, Chen X, Zhu D. Identification of ribosomal RNA methyltransferase gene ermF in Riemerella anatipestifer. Avian Pathol. 2015; 44: 162-168.

11. Chen YP, Tsao MY, Lee SH, Chou CH, Tsai HJ. Prevalence and molecular characterization of chloramphenicol resistance in Riemerella anatipestifer isolated from ducks and geese in Taiwan. Avian Pathol. 2010; 39: 333-338.

12. Chen YP, Lee SH, Chou CH, Tsai HJ. Detection of florfenicol resistance genes in Riemerella anatipestifer isolated from ducks and geese. Vet Microbiol. 2012; 154: 325-331.

13. Yuan J, Liu W, Sun M, Song S, Cai J, Hu S. Complete genome sequence of the pathogenic bacterium Riemerella anatipestifer strain RA-GD. J Bacteriol. 2011; 193: 28962897.

14. Gao M, Jia R, Qiu T, Han M, Wang X. Size-related bacterial diversity and tetracycline resistance gene abundance in the air of concentrated poultry feeding operations. Environ Pollut. 2017; 220: 1342-1348.

15. Zhang P, Shen Z, Zhang C, Song L, Wang B, Shang J, Yue X, Qu Z, Li X, Wu L, Zheng Y, Aditya A, Wang Y, et al. Surveillance of antimicrobial resistance among Escherichia coli from chicken and swine, China, 2008-2015. Vet Microbiol. 2017; 203: 49-55.

16. Hu Q, Zhu Y, Tu J, Yin Y, Wang X, Han X, Ding C, Zhang $\mathrm{B}, \mathrm{Yu}$ S. Identification of the genes involved in Riemerella anatipestifer biofilm formation by random transposon mutagenesis. PLoS One. 2012; 7: e39805.

17. Sievers F, Wilm A, Dineen D, Gibson TJ, Karplus K, Li WZ, Lopez R, McWilliam H, Remmert M, Soding J, Thompson JD, Higgins DG. Fast, scalable generation of high-quality protein multiple sequence alignments using Clustal Omega. Mol Syst Biol. 2011; 7:539.

18. Livak KJ, Schmittgen TD. Analysis of relative gene expression data using real-time quantitative PCR and the 2(-Delta Delta C(T)) Method. Methods. 2001; 25: 402-408.

19. Valdezate S, Vindel A, Echeita A, Baquero F, Canto R. Topoisomerase II and IV quinolone resistance-determining regions in Stenotrophomonas maltophilia clinical isolates with different levels of quinolone susceptibility. Antimicrob Agents Chemother. 2002; 46: 665-671.

20. Fu JF, Tseng YH. Construction of lactose-utilizing Xanthomonas campestris and production of xanthan gum from whey. Appl Environ Microbiol. 1990; 56: 919-923.

21. Lemire BD. Evolution of FOXRED1, an FAD-dependent oxidoreductase necessary for NADH:ubiquinone oxidoreductase (Complex I) assembly. Biochim Biophys Acta. 2015; 1847: 451-457.

22. Speer BS, Salyers AA. Characterization of a novel tetracycline resistance that functions only in aerobically grown Escherichia coli. J Bacteriol. 1988; 170: 1423-1429.

23. Thaker M, Spanogiannopoulos P, Wright GD. The tetracycline resistome. Cell Mol Life Sci. 2010; 67: 419431.

24. Brotz-Oesterhelt H, Brunner NA. How many modes of action should an antibiotic have? Current Opinion in Pharmacology. 2008; 8: 564-573.

25. Olson MW, Ruzin A, Feyfant E, Rush TS 3rd, O'Connell J, Bradford PA. Functional, biophysical, and structural bases forantibacterial activity of tigecycline. Antimicrob Agents Chemother. 2016: 2156-2166.

26. Ruzin A, Visalli MA, Keeney D, Bradford PA. Influence of transcriptional activator RamA on expression of multidrug efflux pump AcrAB and tigecycline susceptibility in Klebsiella pneumoniae. Antimicrob Agents Chemother. 2005; 49: 1017-1022.

27. Visalli MA, Murphy E, Projan SJ, Bradford PA. AcrAB multidrug efflux pump is associated with reduced levels of susceptibility to tigecycline (GAR-936) in Proteus mirabilis. Antimicrob Agents Chemother. 2003; 47: 665-669.

28. Keeney D, Ruzin A, McAleese F, Murphy E, Bradford PA. MarA-mediated overexpression of the AcrAB efflux pump results in decreased susceptibility to tigecycline in Escherichia coli. J Antimicrob Chemother. 2008; 61: 46-53.

29. Salvatore CM, Techasaensiri C, Tagliabue C, Katz K, Leos N, Gomez AM, McCracken GH, Hardy RD. Tigecycline therapy significantly reduces the concentrations of inflammatory pulmonary cytokines and chemokines in a murine model of Mycoplasma pneumoniae pneumonia. Antimicrob Agents Chemother. 2009; 53: 1546-1551.

30. Phillips I, Casewell M, Cox T, De Groot B, Friis C, Jones R, Nightingale C, Preston R, Waddell J. Does the use of antibiotics in food animals pose a risk to human health? A critical review of published data. J Antimicrob Chemother. 2004; 53: 28-52.

31. Silbergeld EK, Graham J, Price LB. Industrial food animal production, antimicrobial resistance, and human health. Annu Rev Public Health. 2008; 29: 151-169.

32. Qin N, Tan X, Jiao Y, Liu L, Zhao W, Yang S, Jia A. RNASeq-based transcriptome analysis of methicillin-resistant Staphylococcus aureus biofilm inhibition by ursolic acid and resveratrol. Sci Rep. 2014; 4: 5467.

33. Munita JM, Arias CA. Mechanisms of Antibiotic 
Resistance. Microbiol Spectr. 2016; 4.

34. Rimler Sa. (1997). Diseases of poultry. (IA: Iowa State University Press Ames).

35. Yang WR, Moore IF, Koteva KP, Bareich DC, Hughes DW, Wright GD. TetX is a flavin-dependent monooxygenase conferring resistance to tetracycline antibiotics. Journal of Biological Chemistry. 2004; 279: 52346-52352.

36. Leelarasamee A. Antibiotics for Resistant Bacteria. Siriraj Med J. 2005; 57: 450-453.

37. Piddock LJV. Multidrug-resistance efflux pumps - not just for resistance. Nature Reviews Microbiology. 2006; 4: 629636.

38. Tan X, Qin N, Wu C, Sheng J, Yang R, Zheng B, Ma Z, Liu L, Peng X, Jia A. Transcriptome analysis of the biofilm formed by methicillin-susceptible Staphylococcus aureus. Sci Rep. 2015; 5: 11997.

39. Collu F, Cascella M. Multidrug resistance and efflux pumps: insights from molecular dynamics simulations. Curr Top Med Chem. 2013; 13: 3165-3183.

40. Zechini B, Versace I. Inhibitors of multidrug resistant efflux systems in bacteria. Recent Pat Antiinfect Drug Discov. 2009; 4: 37-50.

41. Li YF, Jiang HX, Xiang R, Sun N, Zhang YN, Zhao LQ, Gu P, Wang LQ, Zeng ZL. Effects of two efflux pump inhibitors on the drug susceptibility of Riemerella anatipestifer isolates from China. Journal of Integrative Agriculture. 2016; 15: 929-933.

42. Blanco P, Hernando-Amado S, Reales-Calderon JA, Corona F, Lira F, Alcalde-Rico M, Bernardini A, Sanchez MB, Martinez JL. Bacterial Multidrug Efflux Pumps: Much More Than Antibiotic Resistance Determinants. Microorganisms. 2016; 4:14.
43. Pages JM, Amaral L. Mechanisms of drug efflux and strategies to combat them: challenging the efflux pump of Gram-negative bacteria. Biochim Biophys Acta. 2009; 1794: 826-833.

44. Wang X, Ding C, Wang S, Han X, Yu S. Whole-Genome Sequence Analysis and Genome-Wide Virulence Gene Identification of Riemerella anatipestifer Strain Yb2. Appl Environ Microbiol. 2015; 81: 5093-5102.

45. Wang X, Zhu D, Wang M, Cheng A, Jia R, Zhou Y, Chen Z, Luo Q, Liu F, Wang Y, Chen XY. Complete genome sequence of Riemerella anatipestifer reference strain. J Bacteriol. 2012; 194: 3270-3271.

46. Yang W, Moore IF, Koteva KP, Bareich DC, Hughes DW, Wright GD. TetX is a flavin-dependent monooxygenase conferring resistance to tetracycline antibiotics. J Biol Chem. 2004; 279: 52346-52352.

47. Walkiewicz K, Benitez Cardenas AS, Sun C, Bacorn C, Saxer G, Shamoo Y. Small changes in enzyme function can lead to surprisingly large fitness effects during adaptive evolution of antibiotic resistance. Proc Natl Acad Sci U S A. 2012; 109: 21408-21413.

48. Walkiewicz K. Experimental evolution of TetX2: Correlating changes in fitness to their structural and functional origins. 2012; PhD Diss., Rice University.

49. Wang X, Ding C, Han X, Wang S, Yue J, Hou W, Cao S, Zou J, Yu S. Complete Genome Sequence of Riemerella anatipestifer Serotype 1 Strain CH3. Genome Announc. 2015; 3: e01594-14.

50. Chung YT, Hsu W. Functional expression of the bovine herpesvirus 1 alkaline deoxyribonuclease (UL12) in Escherichia coli. Arch Virol. 1996; 141: 2457-2464. 